\title{
Aplicabilidade do digital smile design em reabilitações estéticas: revisão de literatura
}

\author{
Applicability of digital smile design aesthetic \\ rehabilitation: literature review
}

\author{
Natália Rivoli Rossi ${ }^{1}$ (]) \\ Juliana de Freitas Gouveia Silva ${ }^{2}$ (]) \\ Murilo Rocha Rodrigues ${ }^{3}$ (1) \\ Elisa Camargo Kukulka ${ }^{4}$ (1) \\ Manassés Tercio Vieira Grangeiro ${ }^{5}$ (1) \\ Tarcisio José de Arruda Paes Junior 6 (1)
}

\author{
${ }^{1}$ Autora para correspondência. Universidade Estadual Paulista (São José dos Campos). São Paulo, Brasil. ntlrossi@gmail.com \\ 2-6Universidade Estadual Paulista (São José dos Campos). São Paulo, Brasil. freitasgs.juliana@gmail.com, murilorrodrigues@outlook.com, \\ elisakukulka@hotmail.com, terciomanasses@gmail.com, tarcisio.paes@unesp.br
}

RESUMO |OBJETIVO: Revisar a literatura sobre o protocolo de planejamento e diagnóstico digital conhecido como "Digital Smile Design", destacando suas principais vantagens no tocante a reabilitações estéticas em odontologia restauradora. MATERIAL E MÉTODO: Foi realizada busca nas bases de dados da Pubmed, Scielo e Bireme, de 60 artigos, utilizando os termos de busca Dental photography cosmetic dentistry, treatment protocols. Os critérios de inclusão foram: Estudos in vivo, revisões sistemáticas e metanálise, literatura que aborda variáveis em estudo, idioma em português e inglês e artigos publicados no período de 1990 a 2020. Os critérios de exclusão foram: carta ao editor, revisão de literatura e caso clínico. RESULTADOS: O DSD é uma ferramenta de planejamento de tratamento utilizada em odontologia estética interdisciplinar para fortalecer a visão diagnóstica, melhorar a comunicação entre especialistas e a orientação do paciente. $\mathrm{O}$ design do sorriso se refere aos muitos princípios científicos e artísticos que, coletivamente, podem criar um belo sorriso. Esses princípios são estabelecidos por meio de dados coletados dos pacientes como modelos diagnósticos, anamnese e medida de proporções faciais. Por sua vez, a composição estética do sorriso depende de muitos pontos de referência e parâmetros anatômicos para a formação de um conjunto harmônico e belo. CONSIDERAçõES FINAIS: O DSD é uma ferramenta importante para uso em reabilitações estéticas em odontologia restauradora, uma vez que é um instrumento facilitador do diagnóstico, melhora a comunicação entre a equipe multidisciplinar e auxilia na orientação e motivação do paciente, aumentando a previsibilidade dos tratamentos.

PALAVRAS-CHAVE: Fotografia dentária. Odontologia estética. Protocolos de tratamento.
ABSTRACT |OBJECTIVE: To review the literature on the digital planning and diagnosis protocol known as "Digital Smile Design", highlighting its main advantages concerning aesthetic rehabilitation in restorative dentistry. MATERIAL AND METHOD: 60 articles were searched in Pubmed, Scielo, and Bireme databases, using the search terms Dental photography, cosmetic dentistry, treatment protocols. Inclusion criteria were: In vivo studies, systematic reviews and meta-analysis, literature addressing variables under study, language in Portuguese and English, and articles published from 1990 to 2020. Exclusion criteria were: letter to the editor, literature review, and clinical case. RESULTS: DSD is a treatment planning tool used in interdisciplinary aesthetic dentistry to strengthen the diagnostic vision, improve communication between specialists and patient guidance. Smile design refers to the many scientific and artistic principles that, collectively, can create a beautiful smile. These principles are established through data collected from patients as diagnostic models, anamnesis, and measurement of facial proportions. In turn, the aesthetic composition of the smile depends on many points of reference and anatomical parameters for the formation of a harmonious and beautiful set. FINAL CONSIDERATIONS: The DSD is an important tool to be used for aesthetics rehabilitations in restorative dentistry, since it is an instrument that facilitates the diagnosis, improves communication between the multidisciplinary team and assists in the orientation and motivation of the patient, increasing the predictability of treatments.

Keywords: Dental photography. Cosmetic dentistry. Treatment protocols. 


\section{Introdução}

A Odontologia evoluiu para atender as demandas e expectativas mais elevadas em relação à estética dos pacientes na atualidade. Assim, diante da necessidade de desenvolver uma visão e uma habilidade artística, os dentistas precisam ir além das fronteiras da Odontologia tradicional, a fim de se tornarem designers do sorriso ${ }^{1-3}$. O objetivo de todo tratamento estético dental deve ser o de criar um design que se adeque às necessidades funcionais, estéticas e emocionais do paciente. Com efeito, técnicas e materiais modernos podem ser inúteis se o resultado final não atingir as expectativas estéticas do paciente dentro das possibilidades do caso clínico ${ }^{4}$.

Como forma de minimizar possíveis erros em reabilitações de dentes anteriores, foi desenvolvido um protocolo alternativo chamado "Digital Smile Design" (DSD). Esse protocolo tem o intuito de facilitar a visualização dos casos, melhorando o planejamento reabilitador e facilitando a resolução dos tratamentos. $O$ DSD não apenas devolve o dente a sua posição, mas torna possível a visualização de discrepâncias gengivais e da harmonia entre os seis dentes anteriores ${ }^{1-3}$.

A técnica do DSD é acessível pois as fotografias digitais podem ser feitas com equipamentos simples, não exigindo câmeras profissionais ou softwares especiais, podendo as fotos serem trabalhadas em software de apresentação de slides ${ }^{5}$. Através do uso de sofware de apresentação de slides, é possível colocar linhas e desenhos digitais sobre fotos intraorais e da face do paciente, seguindo uma sequência específica para melhor avaliar a relação estética entre dentes, gengiva, sorriso e face, permitindo ao dentista e ao paciente uma melhor compreensão a respeito dos problemas e da elaboração de possíveis soluções através do plano de tratamento ${ }^{6}$. Além disso, há hoje disponível o mercado o próprio sofware do DSD, associando a área odontológica a tecnologia 3D através dos recursos de bioengenharia, a fim de produzir excelentes ferramentas terapêuticas. Este software de processamento tem como objetivo digitalizar todo o fluxo de trabalho de reabilitação, simplificando o trabalho do profissional e facilitando a comunicação com o paciente?

Cada trabalho artístico requer uma visualização inicial, de modo que, na arquitetura, escultura ou pintura, é necessário fazer uso de projetos, esboços ou protótipos, os quais são representações bi ou tridimensionais do resultado final e, depois de terem sido desenvolvidas, irão guiar os processos de construção, desenho e modelagem. Da mesma forma, na Odontologia, todas as necessidades, expectativas, questões funcionais e biológicas dos pacientes devem ser cientificamente incorporadas no desenho estético do tratamento, que deve servir como referência para todo o resto do procedimento ${ }^{\mathbf{8}-12}$.

Diante do exposto, o objetivo do presente estudo foi realizar uma revisão de literatura sobre o protocolo de planejamento e diagnóstico digital conhecido como "Digital Smile Design", destacando os pontos planejamento do tratamento, princípios do design do sorriso, protocolo fotográfico suas principais vantagens no tocante a reabilitações estéticas em odontologia restauradora.

\section{Materiais e métodos}

Foi selecionada a literatura dos ultimos 30 anos, publicados entre 1990 e 2020, através da análise de resumos e abstracts, pelas palavras-chaves buscadas no Mesh: dental photography, cosmetic dentistry, treatment protocols, esthetics, dental prosthesis design, smiling; assim as variaveis do estudo são planejamento do tratamento, principios do design do sorriso, protocolo fotográfico e DSD e Odontologia digital. Os critérios de inclusão foram estudos in vivo, revisões sistemáticas e metanálise, literatura que aborda variáveis em estudo, idioma em português e inglês. Os critérios de exclusão foram: carta ao editor, revisão de literatura e relato de caso. As buscas foram realizadas na base de dados bibliográficos do PubMed, Scielo, Bireme. Além disso, foram utilizadas informações contidas em livros pertinentes ao assunto. 


\section{Revisão de literatura}

\section{Planejamento de tratamento}

Para estabelecer um plano de tratamento reabilitador estético e um correto diagnóstico, é necessário identificar e quantificar quais elementos do sorriso precisam ser corrigidos ou melhorados, e quais devem ser mantidos. Por meio da documentação em vídeo, o paciente se sente menos desconfortável e age mais naturalmente. $\mathrm{O}$ uso de documentação com sorrisos dinâmicos associado ao protocolo de DSD pode trazer diagnósticos mais eficientes, planos de tratamentos mais consistentes, levando a uma sequência de tratamento mais direta e lógica, com redução de riscos e melhora do resultado final2,3,5. Por meio da documentação em vídeo, o paciente se sente menos desconfortável e age mais naturalmente.

O design do sorriso deve ser baseado na compreensão de conceitos macro e micro estéticos, independentemente do sistema usado ${ }^{13,14}$. Esses conceitos permeiam o design do sorriso e são fundamentais no planejamento do tratamento de casos estéticos. Desse modo, o design do sorriso não pode ser isolado de uma abordagem abrangente ao tratamento do paciente, uma vez que um resultado bem-sucedido, saudável e funcional requer uma compreensão da inter-relação entre todas as estruturas orais de apoio, incluindo músculos, ossos, articulações, tecidos gengivais e oclusão. Essa compreensão exige coletar os dados necessários para avaliar adequadamente todas as estruturas do complexo oral ${ }^{15}$.

A coleta de informações dos pacientes, modelos diagnósticos, anamnese, proporções baseadas em evidência científica e conceitos artísticos básicos de beleza são necessários, que deve incluir radiografias dentárias, modelos de diagnóstico montados em articulador, registros fotográficos, exame clínico completo e uma entrevista com o paciente ${ }^{6,12,15-18}$. Além da estética, o componente de função dos dentes anteriores deve ser considerado no planejamento do tratamento. A guia anterior em harmonia com posições articulares saudáveis é fundamental para estabelecer uma disposição oclusal estável15-19.
Sendo assim deve-se realizar uma análise funcional, entendendo quais foram os problemas que geraram as alterações dentárias. Dentre esses problemas, pode-se destacar as discrepâncias oclusais, perda de dimensão vertical de oclusão, más relações ósseas e articulares e hábitos parafuncionais $15,19,20$. A dimensão vertical de oclusão deve ser restabelecida e as respectivas guias de desoclusão, anterior e laterais. Com a oclusão funcional, pode-se planejar a remodelação do segmento anterior.

Como o DSD é um método fotográfico que leva em consideração uma análise dental em relação à face, torna-se possível um resultado mais natural, devolvendo estética e reintegrando o paciente ao meio social, ao elevar sua autoestima e autoconfiança ${ }^{1}$. No entanto, é indispensável que esse protocolo seja associado às demais técnicas como, montagem de modelos de estudo em articulador, análises faciais e estudo de caso e enceramento diagnóstico, já disponíveis e consagradas na literatura odontológica para facilitar o diagnóstico, não sendo viável seu uso isoladamente.

Diante de quadro clínico complexo, em que muitas referências foram perdidas, muitas vezes é difícil estabelecer um ponto de partida para iniciar o tratamento. Nesse momento é importante a análise do caso por uma equipe multidisciplinar para definição do plano de tratamento $1-3,6,2,21,22$.

\section{Princípios do design do sorriso}

O desenho de linhas de referência em fotos extra e intraorais ajuda a equipe a avaliar estética e função, que auxilia na tomada de decisão clínica. Os parâmetros utilizados são linha média e horizontal, linha alta do sorriso, proporção de largura interdental, proporção de altura e largura do incisivo central superior, curva gengival, curva papilar, curva do vermelhão do lábio e curvatura do arco 1,2,5,20 (Figura 1). 
Figura 1. Linhas de referência: linha média e horizontal representada pelas linhas pontilhadas brancas perpendiculares. Linha dos zênites gengivais representada pela linha rosa. Linha alta do sorriso representada pela linha azul. Curva do sorriso representada pela linha amarela. Longo eixo dos dentes representados pelas linhas pretas. Proporção dental representada pelo desenho esquemático dos seis dentes anteriores. Pontos em verde representando os pontos de contato interdentais.

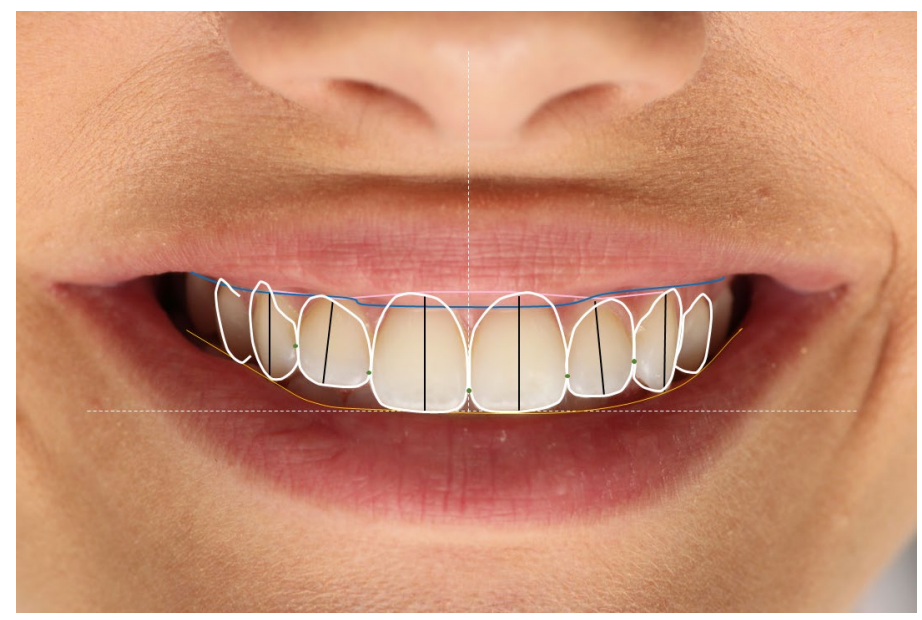

O planejamento deve-se iniciar pela linha mediana, que se refere a uma linha vertical formada pelo contato dos incisivos centrais superiores. A linha mediana deve ser perpendicular ao plano incisal e paralela ou coincidente com a linha mediana da face. A linha de sorriso também é de fundamental importancia para um sorriso agradável. Esta pode ser definida como uma linha imaginária, desenhada ao longo das bordas incisais dos dentes anteriores superiores ${ }^{15}$.

Os princípios do design do sorriso requerem uma integração de conceitos estéticos que harmonizem a estética facial com a composição dento-facial e a composição dental. A composição dento-facial inclui os lábios e o sorriso e como se relacionam com o rosto. Ja a composição dental se refere mais especificamente ao tamanho, forma e posição dos dentes e sua relação com o osso alveolar e os tecidos gengivais. Portanto, o design do sorriso inclui uma avaliação e análise dos tecidos duros e moles do rosto e do sorriso $15,16,19$.

O plano bipupilar relaciona-se com a linha das comissuras e o plano oclusal ${ }^{18}$. A linha bipupilar deve estar paralela à linha do horizonte e perpendicular à linha média da face. Além disso, a linha bipupilar deve estar paralela à linha de comissura e ao plano oclusal ${ }^{23}$.

Outra ferramenta importante para o sucesso de uma reabilitação oral estética é a análise labial. Os lábios desempenham um papel fundamental na medida em que criam os limites da influência do design do sorriso. A compreensão da morfologia dos lábios e de sua mobilidade pode muitas vezes ser útil para atender às expectativas dos pacientes e determinar os critérios para o sucesso da reabilitação. Nesse sentido, existem três aspectos da morfologia labial que devem ser considerados: largura, plenitude e simetria ${ }^{15}$. O volume labial e a simetria dos lábios superiores e inferiores também devem ser documentadas. O volume labial pode ser categorizado como completo, médio ou magro. A simetria labial envolve a aparência de imagem espelhada de cada lábio ao sorrir.

Além disso, a posição dos lábios na posição de repouso deve ser avaliada quanto ao contato labial, bem como quanto à amplitude de mobilidade labial ao sorrir. Esses dois fatores determinam o quanto de estrutura dentária e de tecido gengival será revelado ao comparar as posições de repouso e sorriso completo. Avaliar esse aspecto dentário-facial pode ser útil para analisar e determinar as modalidades de tratamento necessárias para melhorar o sorriso. A avaliação labial também é útil quando se considera a expectativa do paciente e, o mais importante, revela assimetrias e defeitos de dentes e tecidos ${ }^{15}$.

A área entre as comissuras labiais durante a formação do sorriso e as superfícies vestibulares dos dentes da maxila (particularmente os pré-molares e molares) formam um espaço conhecido como corredor bucal. 
Um corredor bucal adequado e simétrico é um elemento importante de um sorriso estético ${ }^{15,20}$.

Outra característica do design do sorriso que é muito significativa, mas frequentemente ignorada, é a saúde, a simetria e a arquitetura dos tecidos gengivais. Esses tecidos enquadram os dentes e aumentam a simetria do sorriso. A saúde, cor e textura dos tecidos gengivais são primordiais para o sucesso a longo prazo e o valor estético do tratamento 22 .

Há várias outras considerações a serem feitas ao se tentar restabelecer a altura normal dos dentes, dependendo da etiologia do desgaste dentario. Discrepâncias oclusais, diminuição da dimensão vertical, desgaste anterior, más relações ósseas e articulares e hábitos parafuncionais podem ser considerados fatores que alteram a estética do sorriso. Por outro lado, o diagnóstico correto leva às opções de tratamento mais adequadas para sucesso e estabilidade a longo prazo.

O desenho das linhas de referência nas fotografias podem ser sobrepostas ao exame tomográfico e ao modelo digital do paciente no computador. A conjunção dessas referências permite ao cirurgião dentista a transposição para um projeto 3D, seja por meio analógico (enceramento tradicional) ou digital (enceramento digital 3D em software) ${ }^{1}$.

\section{Protocolo fotográfico}

Apesar de muitos estudos avaliarem o sorriso estático, é difícil capturar uma foto ideal. Nesse sentido, vídeos podem ser usados para a captura do movimento e da integração dinâmica dos dentes, gengiva, lábios e face. Desse modo, a percepção da estética é diferente entre fotos estáticas e dinâmicas. A fotografia pode criar ilusões que às vezes transforma algo agradável em algo desagradável, ou vice-versa ${ }^{1,2,5}$.

O uso de vídeos aumentam a chance de encontrar um sorriso espontâneo, de forma que a criação de um protocolo de vídeo é vantajosa para uma melhor análise, comunicação entre a equipe, planejamento do tratamento e educação do paciente ${ }^{24}$. Para isso, são realizados oito vídeos, sendo quatro deles para o processo técnico de design do sorriso e os demais vídeos complementares. Dentre os vídeos técnicos, estão: vídeo facial frontal, com e sem afastador, vídeo facial de perfil, vídeo 12 horas e vídeo anterior oclusal (Figuras 2-5) .

Figura 2. Vídeo facial frontal sem e com afastador

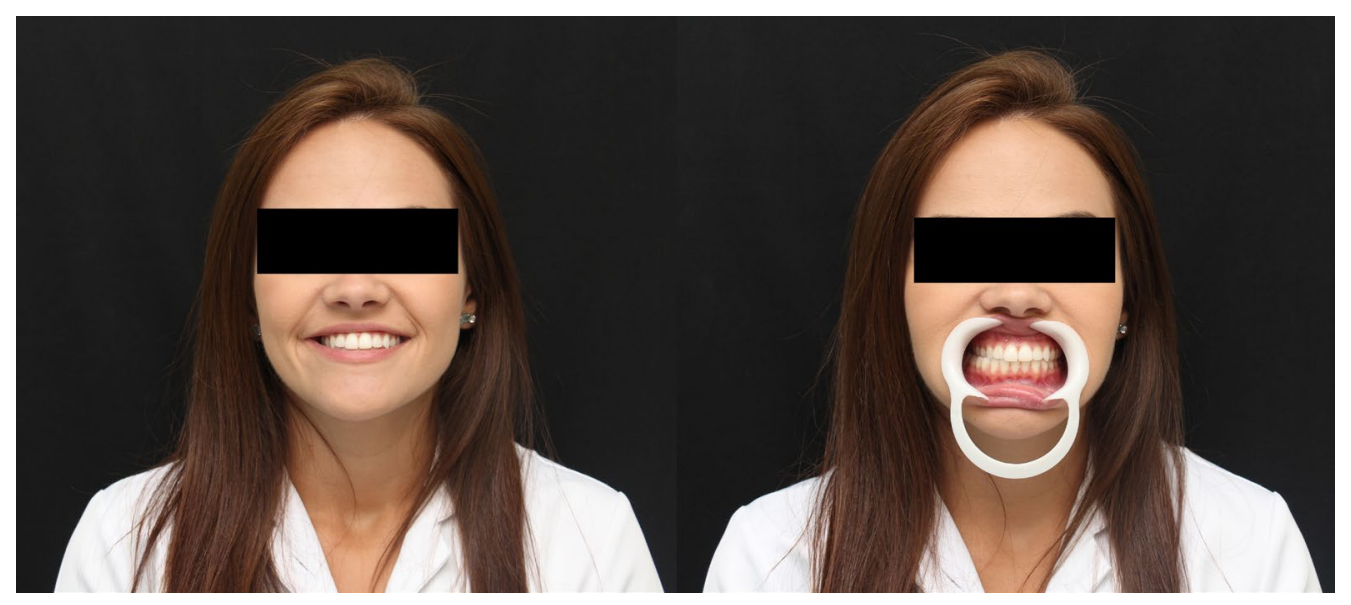


Figura 3. Vídeo facial de perfil

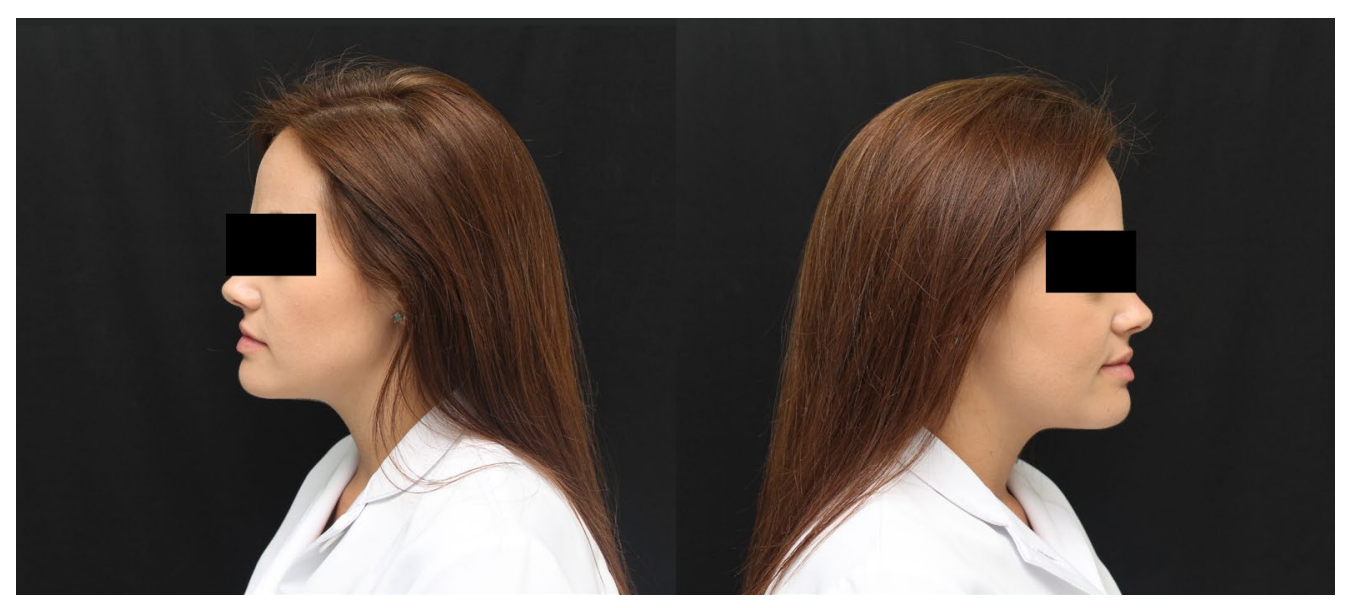

Figura 4. Vídeo 12 horas

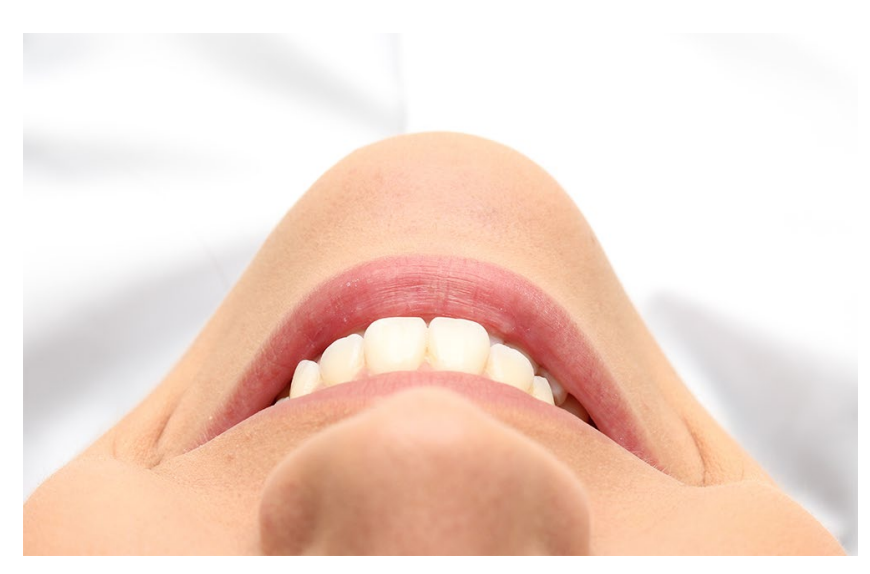

Figura 5. Vídeo anterior oclusal

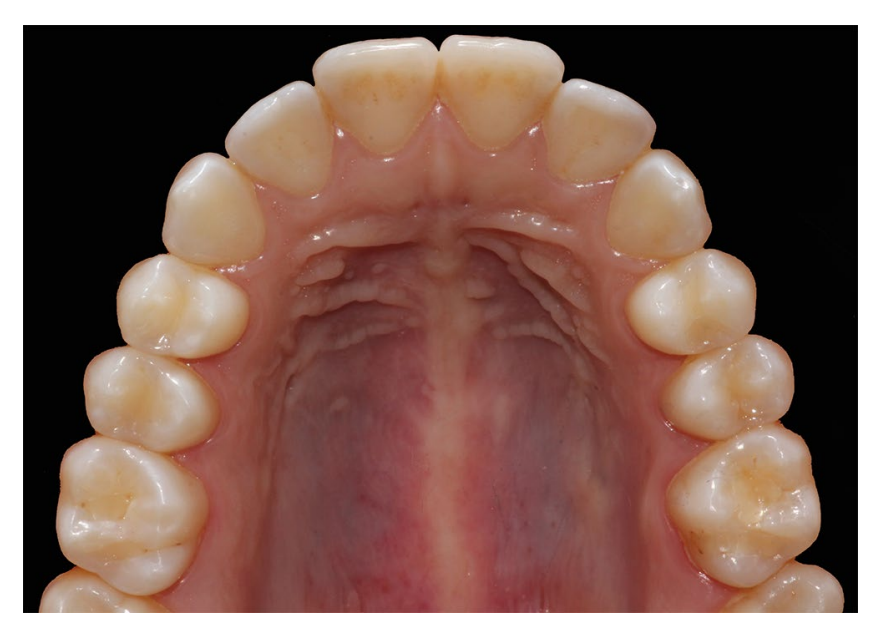


Já os vídeos complementares são: entrevista facial, close up na fonética (sons como f, v, s), movimentos funcionais. Após a confecção dos vídeos, capturando o momento ideal do sorriso, pode-se realizar o desenho das linhas. Esse projeto 2D pode ser transferido para o modelo de trabalho utilizando uma régua digital no computador e um compasso no modelo (Figura 6) ${ }^{5}$.

Figura 6. Projeto 2D de design do sorriso com régua digital

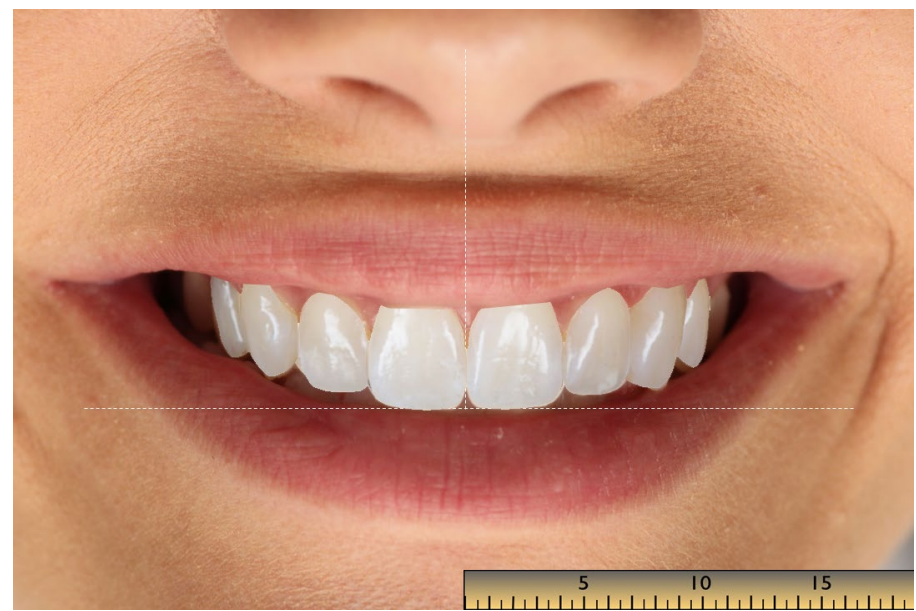

Os vídeos, que devem ser mostrados aos pacientes, são um caminho eficiente para a revelação dos problemas e das possíveis soluções. Criar um link emocional entre o paciente e o possível tratamento antes de discutir as questões técnicas e financeiras envolvidas é um fator chave para melhorar o processo de orientação e de motivação do paciente, aumentando a aceitação do caso. Dessa forma, mostrar vídeos do mock up motivacional é o método mais fácil e simples para gerar vínculo do paciente com o tratamento ${ }^{5}$.

Os vídeos reduzem as chances de erros, mas sabe-se que as fotos ainda são muito utilizadas pelos clínicos. Em relação as fotos, é importante salientar que uma fotografia incorreta pode levar a uma imagem distorcida que, por sua vez, pode resultar em proporções incorretas. Assim, fica claro que qualquer tipo de planejamento de sorriso digital é apenas uma ferramenta de assistência. Após o planejamento, uma unidade de teste de restauração provisória (protótipo) proporcionará ao paciente e ao clínico a antevisão do resultado final17.

\section{DSD e a Odontologia digital}

A tecnologia CAD-CAM permite uma melhor comunicação e integração entre o técnico de laboratório e cirurgião-dentista, facilitando a verificação do espaço necessário para a futura restauração durante a fase de enceramento diagnóstico digital. A foto facial ou o escaneamento facial 3D podem ser combinados com o escaneamento intraoral, sobrepondo os arquivos gerados. Isto permite a visualização em tempo real da estética facial e possibilita quaisquer alterações no plano de tratamento, o que não era possível no formato analógico tradicional $\$ 2,25$.

Para gerar os modelos digitais, necessários na realização dos planejamentos e enceramentos digitais, pode-se utilizar escaners intraorais. Os escaneamentos realizados pelo cirurgião-dentista e os modelos digitais gerados trazem agilidade aos procedimentos reabilitadores. A opção de se trabalhar "chairside" baseia-se na obtenção de modelos digitais através do escaneamento do paciente. A partir deste ponto, como já dito, é possível realizar o enceramento digital, que torna ainda mais previsível e fiel o planejamento proposto ao caso clínico. É ainda melhor quando combinado com as fotos do paciente. A sobreposição das fotos, dos modelos digitais, e do enceramento diagnóstico, gera peças protéticas em 3D que podem ser fresadas pela tecnologia CAD/CAM. Pode-se também fazer uso da impressão 3D, onde o enceramento digital é impresso gerando um modelo protótipo do sorriso, sendo este transferido a boca do paciente por guia de silicone para realização do 
mock-up para prova estética e funcional das futuras próteses ${ }^{26}$. Somado a isso, através do software de processamento do DSD, a digitalização completa do fluxo de trabalho de reabilitação pode ser associada a todos esses recursos, facilitando ainda mais a vida do profissional clínico ${ }^{7}$. É extremamente importante salientar que a fotografia e a captura de detalhes é importante para exame, diagnóstico, plano de tratamento e para ampliar a previsibilidade dos tratamentos, sendo também crucial para o registro legal da situação clínica $1,2,5,5,6,13,15,21,27$.

\section{Considerações finais}

O DSD é uma ferramenta importante para solucionar problemas estéticos visíveis nos pacientes. Além de visualizar o possível resutado do tratamento, o DSD melhora o diagnóstico e o planejamento do tratamento, sendo assim, uma ferramenta importante para uso em reabilitações estéticas em odontologia restauradora.

\section{Contribuições dos autores}

Rossi NR escreveu e corrigiu o artigo, editou as imagens. Silva JFG e Kukulka EC escreveram o artigo. Rodrigues MR escreveu o artigo e fotografou a modelo. Grangeiro MTV escreveu e corrigiu o artigo. Paes Junior TJA - idealizou e supervisionou a execução do artigo.

\section{Conflitos de interesses}

Nenhum conflito financeiro, legal ou político envolvendo terceiros (governo, empresas e fundações privadas, etc.) foi declarado para nenhum aspecto do trabalho submetido (incluindo, mas não se limitando a subvenções e financiamentos, participação em conselho consultivo, desenho de estudo, preparação de manuscrito, análise estatística, etc.).

\section{Referências}

1. Coachman C, Calamita MA, Sesma N. Dynamic documentation of the smile and the 2D/3D Digital Smile Design process. Int J Periodontics Restorative Dent. 2017;37(2):183-93. doi: $10.11607 /$ prd.2911

2. Coachman C, Paravina RD. Digitally enhanced esthetic dentistry - From treatment planning to quality control. J Esthet Restor Dent. 2016;28(Suppl 1):S3-4. doi: 10.1111/jerd.12205
3. Jafri Z, Ahmad N, Sawai M, Sultan N, Bhardwaj A. Digital Smile Design-An innovative tool in aesthetic dentistry. J Oral Biol Craniofacial Res. 2020;10(2):194-198. doi: 10.1016/j. jobcr.2020.04.010

4. Omar D, Duarte C. The application of parameters for comprehensive smile esthetics by digital smile design programs: A review of literature. Saudi Dent J. 2018;30(1):7-12. doi: 10.1016/j. sdentj.2017.09.001

5. Coachman C, Calamita M. Digital smile design: a tool for treatment planning and communication in aesthetic dentistry. Quintessence Dent Technol. 2012;35:103-111.

6. Dawson PE. Functional occlusion: From TMJ to smile design. 1.ed. St Louis: Mosby; 2006.

7. Cervino G, Fiorillo L, Arzukanyan AV, Spagnuolo G, Cicciù M. Dental restorative digital workflow: Digital smile design from aesthetic to function. Dent J. 2019;7(2):30. doi: 10.3390/dj7020030

8. Spear FM. The maxillary central incisor edge: a key to esthetic and functional treatment planning. Compend Contin Educ Dent. 1999;20(6):512-6.

9. Spear FM, Kokich VC, Mathews DP. Interdisciplinary management of anterior dental esthetics. J Am Dent Assoc. 2006;137(2):160-9. doi: 10.14219/jada.archive.2006.0140

10. Goldstein RE. Esthetics in dentistry: principles, communication, treatment methods. 2.ed. Ontario: BC Decker; 1998.

11. Cohen M. Interdisciplinary treatment planning. Principles, Design, Implementation. 1.ed. Quintessence Publishing Co Inc. Hannover Park; 2008.

12. Rifkin R. Facial analysis: a comprehensive approach to treatment planning in aesthetic dentistry. Pract Periodontics Aesthet Dent. 2000;12(9):865-71.

13. Arias DM, Trushkowsky RD, Brea LM, David SB. Treatment of the Patient with Gummy Smile in Conjunction with Digital Smile Approach. Dent Clin North Am. 2015;59(3):703-16. doi: 10.1016/j. cden.2015.03.007

14. McLaren EA, Goldstein RE. The Photoshop Smile Design technique. Compend Contin Educ Dent. 2018;39(5):e17-20.

15. Davis NC. Smile Design. Dent Clin North Am. 2007;51(2):299318. doi: 10.1016/j.cden.2006.12.006

16. Coachman C, Salama M, Garber D, Calamita M, Salama $\mathrm{H}$, Cabral G. Prosthetic gingival reconstruction in fixed partial restorations. Part 3: laboratory procedures and maintenance. Int J Periodontics Restorative Dent. 2010;30(1):19-29.

17. Zanardi PR, Zanardi RLR, Stegun RC, Sesma N, Costa B, Laganá DC. The Use of the Digital Smile Design Concept as an Auxiliary Tool in Aesthetic Rehabilitation : A Case Report. Open Dent J. 2016;10:28-34. doi: $10.2174 / 1874210601610010028$ 
18. Rufenacht CR. Fundamentals of esthetics. 1.ed. Chicago: Quintessence Publishing Co Inc; 1990.

19. Calamia JR, Levine JB, Lipp M, Cisneros G, Wolff MS. Smile design and treatment planning with the help of a comprehensive esthetic evaluation form. Dent Clin North Am. 2011;55(2):187-209. doi: 10.1016/j.cden.2011.01.012

20. Mondelli J, Souza Júnior MS, Mondelli R. Estética e cosmetica em dentistica restauradora. In: Médicas A, editor. Atualizacao Na Clinica Odontologica: a Pratica da Clinica Geral. 1st ed. 1996. p. 93-129.

21. Paolucci B, Calamita M, Coachman C, Gürel G, Shayder A, Hallawell P. Visagism: the art of dental composition. Quintessence Dent Technol. 2012;35:187-200.

22. Coachman C, Dooren EV. An integrated cosmetic treatment plan: soft tissue management and metal-free restoration. Int Dent - African Ed. 2012;2(3):5-9.
23. Rufenacht CR. Principles of Esthetic Integration. 1.ed. Chicago: Quintessence Publishing Co; 2000.

24. Stanley M, Paz AG, Miguel I, Coachman C, Miguel I. Fully digital workflow, integrating dental scan, smile design and CAD-CAM: Case report. BMC Oral Health. 2018;18:134. doi: 10.1186/s12903018-0597-0

25. Silva BP, Stanley K, Gardee J. Laminate veneers: Preplanning and treatment using digital guided tooth preparation. J Esthet Restor Dent. 2020;32(2):150-60. doi: 10.1111/jerd.12571

26. Coachman C, Georg R, Bohner L, Rigo LC, Sesma N. Chairside 3D digital design and trial restoration workflow. J Prosthet Dent. 2020;19:1-7. doi: 10.1016/j.prosdent.2019.10.015

27. Casaglia A, Dominicis P, Arcuri L, Gargari M, Ottria L. Dental photography today. Part 1: Basic concepts. Oral Implant. 2016;8(4):122-9. doi: 10.11138/orl/2015.8.4.122 